\title{
Analysis of the Modal Behavior of an Antiguide Diode Laser Array with Talbot Filter
}

Pieter D. Van Eijk, Member, IEEE, Muriel Reglat, Georges Vassilieff, Gijs J. M. Krijnen, Student Member, IEEE, Alfred Driessen, and Anton J. Mouthaan, Member, IEEE

\begin{abstract}
An analysis of the filtering of the array modes in a resonant optical waveguide (ROW) array of antiguides by a diffractive spatial filter, a so-called Talbot filter, is presented. A dispersion relation is derived for the array modes enabling the calculation of the field distribution. The filtering is analyzed by calculating the field propagation of each array mode in an array structure with a Talbot filter by means of the beam-propagation method. It is shown that in the presented array structure only the resonant in-phase mode will lase.
\end{abstract}

\section{INTRODUCTION}

A PPLICATIONS of diode laser arrays in optical computing and free-space communication require high powers in stable, diffraction-limited beams [1]. Single inphase array-mode operation is a requisite since array operation of a mixture of the in-phase mode and other array modes will give rise to a nondiffraction limited output. Therefore, the other modes have to be suppressed.

Recently, an ROW array of antiguides with a monolithically integrated Talbot filter to suppress the out-of-phase mode has been reported [2]-[4]. The effect of the Talbot filter on the out-of-phase, the in-phase mode, and it's adjacent modes has been analyzed by calculating a field overlap integral [5]. Up to now, however, to our knowledge, no analysis of the discrimination of the Talbot filter against the array modes has been made by actually calculating the field propagation of each array mode in a structure with a monolithically integrated Talbot filter.

\section{THEORY}

In order to find the array modes in the array of $L$ antiguides ( $L$ is odd in our case) the problem has been simplified to a two-dimensional one by representing the array by a multilayered structure of $N$ layers $(N=2 L+1)$ with an alternating complex refractive index $\bar{n}_{1}, \bar{n}_{0}, \bar{n}_{1}$, $\cdots, \bar{n}_{0}, \bar{n}_{1}\left(\operatorname{Re}\left\{\bar{n}_{0}\right\}<\operatorname{Re}\left\{\bar{n}_{1}\right\}\right)$ [2]. Fig. 1 displays the multilayered structure in which the indexes $\bar{n}_{0}$ and $\bar{n}_{1}$

Manuscript received July 16, 1990; revised November 13, 1990

P. D. Van Eijk, G. J. M. Krijnen, A. Driessen, and A. J. Mouthaan are with the University of Twente, Faculties of Electrical Engineering and Applied Physics, 7500 AE Enschede, The Netherlands.

M. Reglat and G. Vassilieff are with the Laboratoire d'Automatique et d'Analyse des Systèmes du C.N.R.S., Equipe C.E.L., 31077 Toulouse Cédex, France.

IEEE Log Number 9042362. are the effective indexes corresponding with the antiguide core and cladding, respectively. The antiguide core has width $d$ and the cladding, the so-called interelement region, has width $s$.

An ROW array of antiguides has a resonant in-phase array mode which is characterized by strong interelement coupling resulting in a uniform intensity profile. The interelement spacing needed for a resonant operation of the in-phase mode, TE or TM polarized, can be found easily by using the following equation [2]:

$$
\begin{gathered}
s_{\mathrm{res}} \approx \frac{L \lambda}{2 \sqrt{\operatorname{Re}\left\{n_{1}\right\}^{2}-\operatorname{Re}\left\{n_{0}\right\}^{2}+(\lambda / 2 d)^{2}}}, \\
L=1,3,5, \cdots
\end{gathered}
$$

in which $\lambda$ is the wavelength of the laser array. Single, resonant in-phase array-mode operation will not only result in a diffraction-limited but also in a strong coherent output.

\section{A. The Dispersion Relation}

Field equations have been derived for both TM- and TE-polarized modes in the multilayered structure shown in Fig. 1. The solution of the magnetic (TM) and the electric (TE) fields in the $y$ direction is denoted by the following set of equations in which the origin of our coordinate system has been chosen at the center of the array (the term $\exp [j(\omega t-\beta z)]$ has been omitted for simplicity):

$$
\begin{aligned}
& A_{-N}=B_{-N} \exp \left[j h_{N}\left(x+t_{n}\right)\right] \quad x<-t_{n} \\
& \vdots \quad \vdots \quad \vdots \\
& A_{-i}=B_{-i} \cos \left[h_{i}\left(x+t_{i}\right)-\phi_{-(i-1)}\right] \quad-t_{i+1}<x<-t_{i} \\
& \text { : } \quad \vdots \quad \vdots \\
& A_{0}=B_{0} \cos \left[h_{0} x-\phi_{0}\right] \quad t_{-1}<x<t_{1} \\
& A_{i}=B_{i} \quad \cos \left[h_{i}\left(x-t_{i}\right)-\phi_{(i-1)}\right] \quad t_{i}<x<t_{i+1} \\
& A_{N}=B_{N} \quad \exp \left[-j h_{N}\left(x-t_{n}\right)-\phi_{(i-1)}\right] \quad t_{N}<\quad x
\end{aligned}
$$




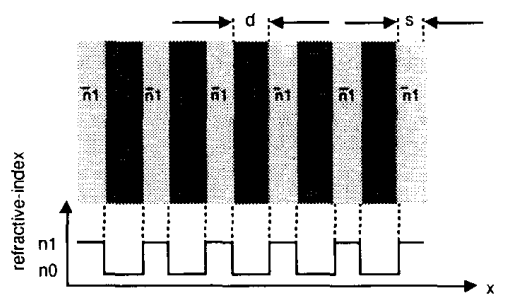

$x=$ lateral $y=$ tranversal

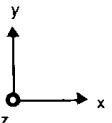

Fig. 1. Multilayered structure with alternating layers of refractive index $\bar{n}_{0}$ and $\bar{n}_{1}$. The antiguide core width and the interelement spacing are de noted by $\mathrm{d}$ and $\mathrm{s}$, respectively. (Only five antiguides shown.)

in which

$$
\begin{aligned}
A_{i} & =E_{y}(x) \text { for TE polarized modes } \\
A_{i} & =H_{y}(x) \text { for TM polarized modes. }
\end{aligned}
$$

The constants $B_{i}$ follow from the continuity of $A_{i}$ at the interfaces. The lateral propagation vector $h_{i}$ is defined as

$$
h_{i}=k_{0} \sqrt{\bar{n}_{x}^{2}-\bar{n}_{\mathrm{eff}}^{2}}, \quad x \in\{0,1\}
$$

in which $k_{0}$ equals $2 \pi / \lambda_{0}, \lambda_{0}$ being the free-space wavelength. The complex refractive indexes $\bar{n}_{0}$ and $\bar{n}_{1}$ are written as

$$
\bar{n}_{x}=n_{x}+j \frac{g_{x}}{2 k_{0}}, \quad x \in\{0,1\}
$$

where $g_{x}>0$ represents gain and $g_{x}<0$ loss. The effective index $\bar{n}_{\text {eff }}$ of the array mode(s) is defined as

$$
\bar{n}_{\mathrm{eff}}=n_{\mathrm{eff}}+j \frac{g_{\mathrm{eff}}}{2 k_{0}}
$$

in which $g_{\text {eff }}$ represents the modal gain. The constants $t_{i}$ are defined as

$$
\begin{gathered}
t_{i}=\frac{(i-1)}{2}(s+d)+\frac{d}{2} \quad i=\text { odd, } 1 \leq|i| \leq N \\
t_{i}=\frac{i}{2}(s+d)-\frac{d}{2} \\
\quad i=\text { even, } 2 \leq|i| \leq(N-1)
\end{gathered}
$$

The phase terms $\phi_{i}$, which follow from the continuity of the $H_{z}\left(E_{y}\right)$ components for TE (TM) modes at the interfaces, are written as

$$
\begin{aligned}
& \phi_{-(N-1)}=\arctan \left\{-j \frac{h_{N}}{h_{N-1}}\left[\frac{\bar{n}_{N-1}}{\bar{n}_{N}}\right]^{p}\right\}-h_{N-1} d \\
& \vdots \\
& \phi_{-i}=\arctan \left\{\tan \left[\phi_{-(i+1)}\right] \frac{h_{i+1}}{h_{i}}\left[\frac{\bar{n}_{i}}{\bar{n}_{i+1}}\right]^{p}\right\}-h_{i} l_{i} \\
& \vdots \\
& \phi_{0}=\frac{m \pi}{2}
\end{aligned}
$$

$$
\begin{aligned}
\phi_{i} & =-\arctan \left\{\tan \left[-\phi_{(i+1)}\right] \frac{h_{i+1}}{h_{i}}\left[\frac{\bar{n}_{i}}{\bar{n}_{i+1}}\right]^{p}\right\}+h_{i} l_{i} \\
\vdots & (8 \mathrm{~d}) \\
\phi_{N-1} & =-\arctan \left\{j \frac{h_{N}}{h_{N-1}}\left[\frac{\bar{n}_{N-1}}{\bar{n}_{N}}\right]^{p}\right\}+h_{N-1} d
\end{aligned}
$$

in which

$$
\begin{aligned}
& p=0 \text { for TE polarization } \\
& p=2 \text { for TM polarization }
\end{aligned}
$$

and where

$$
\begin{aligned}
& l_{i}=d \text { if } i \text { is even } \\
& l_{i}=s \text { if } i \text { is odd. }
\end{aligned}
$$

Based on the continuity of the fields a dispersion relation for TE and TM polarization can be derived from (2)-(10)

$$
\begin{aligned}
\bar{n}_{0}^{2}-\bar{n}_{\text {eff }}^{2}= & {\left[\frac{1}{d k_{0}}\right]^{2}\left\{2 \operatorname { a r c t a n } \left[\tan \left(-\phi_{1}\right)\left(\frac{\bar{n}_{0}}{\bar{n}_{1}}\right)^{p}\right.\right.} \\
& \left.\left.\cdot \sqrt{\frac{\bar{n}_{1}^{2}-\bar{n}_{\text {eff }}^{2}}{\bar{n}_{0}^{2}-\bar{n}_{\text {eff }}^{2}}}\right]+m \Pi\right\}^{2} .
\end{aligned}
$$

By varying the integer $m$, which equals 0,1 or 2 , all the array modes can be found. For an array of $L$ antiguides more than $L$ modes exist, however, the first $L$ modes have a much higher modal gain than the remaining ones and those will only be considered here.

\section{B. The Talbot Filter}

Wilcox et al. [6] showed that a set of in-phase Gaussian curves, representing the in-phase mode, will shift laterally by $(d+s) / 2$ after having propagated a half-Talbot distance through a homogeneous medium. This half-Talbot distance $Z_{T} / 2$ is defined as

$$
Z_{T} / 2=\frac{\Lambda^{2} n}{\lambda}
$$

in which $n$ is the real part of the refractive index of the homogeneous medium in the Talbot filter, and $\Lambda$ being the center-to-center array element spacing $(s+d)$. It has also been shown [6] that a set of out-of-phase Gaussian curves, representing the out-of-phase mode will mirror at half-Talbot distance. Mawst et al. [3] used an array structure with two noncolinear sets of antiguides separated by a half-Talbot distance, as depicted in Fig. 2, to suppress the out-of-phase mode. (The second set of antiguides has an extra element since the Talbot filtering introduces an extra "Gaussian" for the in-phase mode.) At the end of the Talbot filter a major part of the intensity of this mode is situated in the interelement regions, characterized by high losses, resulting in the suppression of the out-of- 


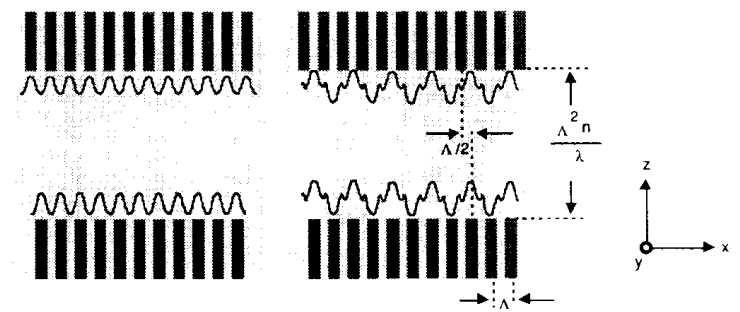

Fig. 2. Schematic representation of two noncolinear sets of antiguides separated by the half-Talbot distance; field amplitudes of the in- and out-ofphase mode.

TABLE I

Real Parts of the Effective Indexes and Modal Gains of the Eleven Array Modes. Te AND TM POLARIZED

\begin{tabular}{|c|c|c|c|c|c|c|}
\hline \multirow{3}{*}{$\begin{array}{l}\text { Array } \\
\text { Mode } \\
\text { Number }\end{array}$} & \multirow{2}{*}{\multicolumn{2}{|c|}{$\frac{\text { Dispersion Relation }}{\text { TM Polarization }}$}} & \multicolumn{4}{|c|}{ Slab-Solving Program } \\
\hline & & & \multicolumn{2}{|c|}{ TM Polarization } & \multicolumn{2}{|c|}{ TE Polarization } \\
\hline & $n_{\text {eff }}$ & $\underset{\left[\mathrm{cm}^{-1}\right\rceil}{g_{\text {eff }}}$ & $n_{\text {eff }}$ & $\underset{\left[\mathrm{cm}^{-1}\right]}{g_{\text {eft }}}$ & $n_{\text {eff }}$ & $\underset{\left[\mathrm{cm}^{-1}\right]}{g_{\mathrm{eff}}}$ \\
\hline 1 & 3.412000 & 58.594 & 3.412000 & 58.533 & 3.412000 & 59.033 \\
\hline 2 & 3.412412 & 31.562 & 3.412411 & 31.486 & 3.412408 & 31.270 \\
\hline 3 & 3.412779 & 33.900 & 3.412781 & 33.887 & 3.412774 & 33.608 \\
\hline 4 & 3.413106 & 36.238 & $3.413 \quad 103$ & 36.214 & 3.413100 & 36.092 \\
\hline 5 & 3.413397 & 38.576 & 3.413399 & 38.528 & 3.413389 & 38.284 \\
\hline 6 & 3.413650 & 40.622 & 3.413647 & 40.541 & 3.413641 & 40.329 \\
\hline 7 & 3.413867 & 42.375 & 3.413863 & 42.420 & 3.413857 & 42.083 \\
\hline 8 & 3.414044 & 43.982 & 3.414043 & 43.900 & 3.414033 & 43.544 \\
\hline 9 & 3.414183 & 45.151 & 3.414183 & 45.086 & 3.414172 & 44.713 \\
\hline 10 & 3.414283 & 46.028 & 3.414283 & 46.026 & 3.414271 & 45.590 \\
\hline 11 & 3.414343 & 46.612 & 3.414341 & 46.494 & 3.414331 & 46.174 \\
\hline
\end{tabular}

phase mode. However, an overall analysis of the effect of the Talbot filter on all the array modes has not yet been reported.

\section{Computational Results}

An array of eleven antiguides, emitting at $860 \mathrm{~nm}$, has been analyzed. The refractive indexes of the antiguides (core width $3 \mu \mathrm{m}$ ) $n_{0}$, and of the interelement regions $n_{1}$, have been taken as 3.415 and 3.465 , respectively. Furthermore, the gain $g_{0}$ in the antiguides was set at $75 \mathrm{~cm}^{-1}$, while the gain $g_{1}$ in the interelement regions was chosen as $-75 \mathrm{~cm}^{-1}$, representing losses. The interelement spacing needed for a resonant operation of the in-phase mode can be found using (1) ( $L$ has been taken as 3$): s_{\text {res }}=$ $2.14 \mu \mathrm{m}$. The length of both sets of antiguides (Fig. 2) was taken as $50 \mu \mathrm{m}$. The sets were separated by a uniform area with a refractive index equal to $n_{1}$ and with losses of $15 \mathrm{~cm}^{-1}$. Using (12) we find a half-Talbot distance of $106.4 \mu \mathrm{m}$.

\section{A. Array-Mode Calculation}

The effective indexes of the eleven array modes were calculated by solving the dispersion relation (11) using a standard mathematical function [9] based on Secant's method. Table I shows the real part $n_{\text {eff }}$ of the effective

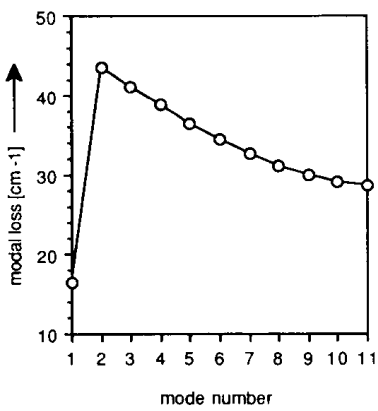

Fig. 3. Modal losses ( $\left.g_{\mathrm{eff}}-g_{0}\right)$ of the eleven array modes (TM polarized) in an ROW array of antiguides.

indexes and the modal gain of the TM-polarized array modes, which are within a small interval of $n_{\text {eff. }}$ The solutions found by means of the dispersion relation were in good agreement with those found by an ordinary slabsolving program using a $2 \mathrm{D}$-Newton-Rhapson iteration in the complex $B$-plane starting at the values found by the dispersion relation, as can be inferred from Table I. It also shows that the effective indexes for TE- and TM-polarized modes are in close proximity. The intensity profiles of modes 1, 2, and 11, TM polarized, are depicted in Figs. 


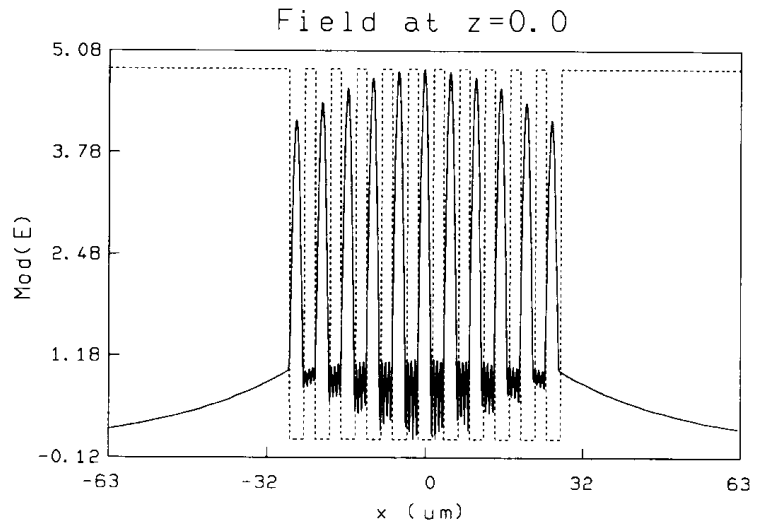

(a)

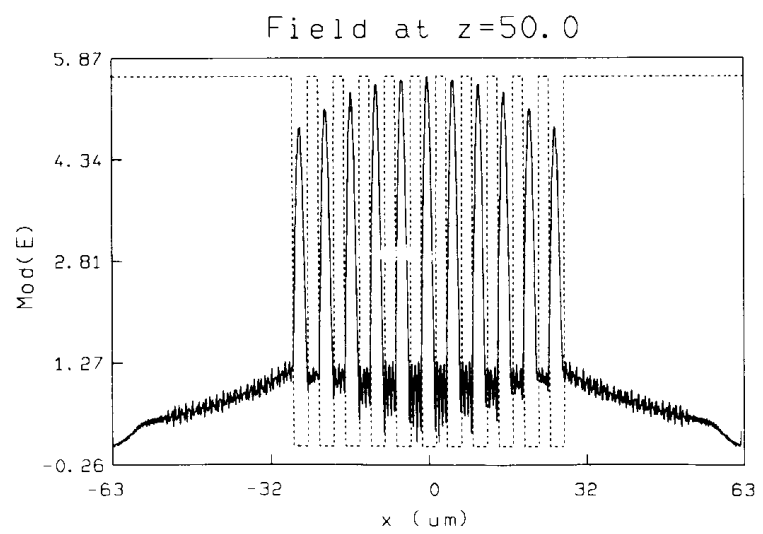

(b)

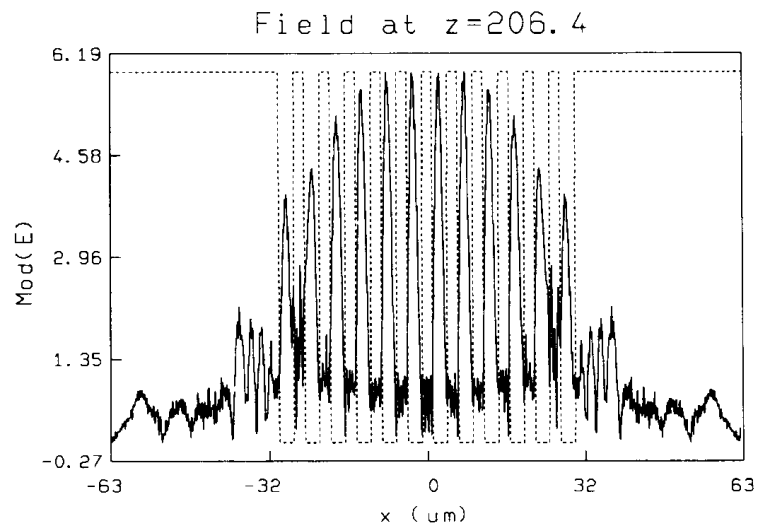

(c)

Fig. 4. Intensity profiles of (a) mode 1 at $z=0 \mu \mathrm{m}$, (b) mode 1 at $z=50$ $\mu \mathrm{m}$, and (c) mode 1 at $z=206.4 \mu \mathrm{m}$. (The dashed lines represent the real refractive-mode profile.)

4(a), 5(a), and 6(a), respectively. The in-phase mode, mode 1 , operates close to resonance since its intensity profile is nearly uniform.

Fig. 3 depicts the modal loss $\left(g_{\text {eff }}-g_{0}\right)$ of the eleven array modes (TM polarized). It can be inferred from this

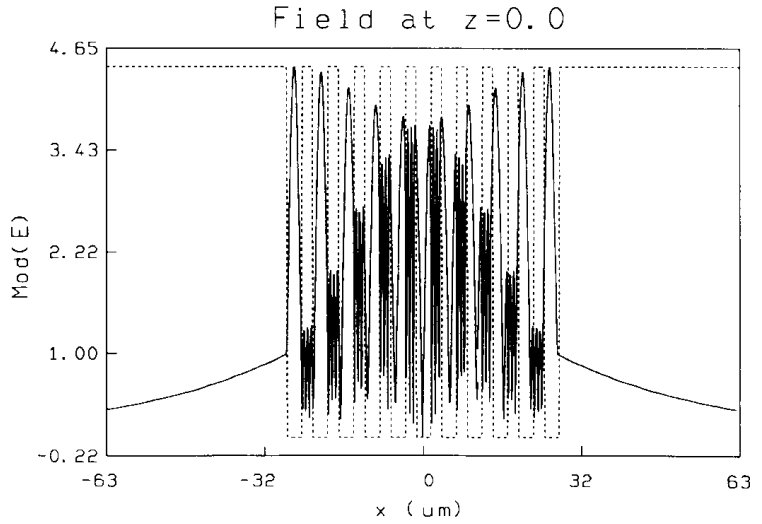

(a)

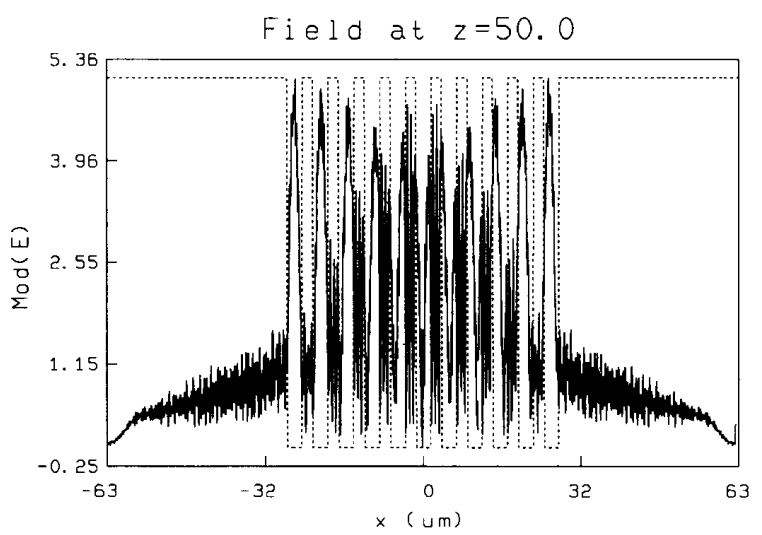

(b)

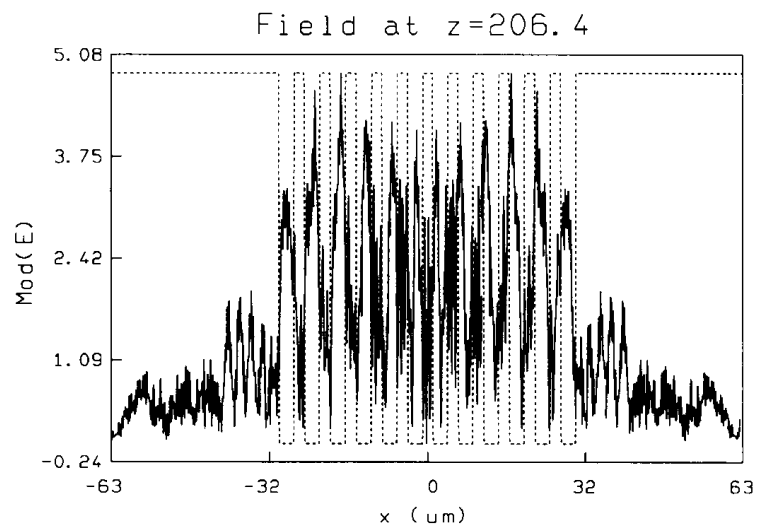

(c)

Fig. 5. Intensity profiles of (a) mode 2 at $z=0 \mu \mathrm{m}$, (b) mode 2 at $z=50$ $\mu \mathrm{m}$, and (c) mode 2 at $z=206.4 \mu \mathrm{m}$. (The dashed lines represent the real refractive-index profile.)

figure and from Table I that the in-phase mode, mode 1, is favorite to lase since it has the highest modal gain. The other array modes can emerge with increasing drive level, resulting in a nondiffraction limited output. Therefore, these modes have to be suppressed. 


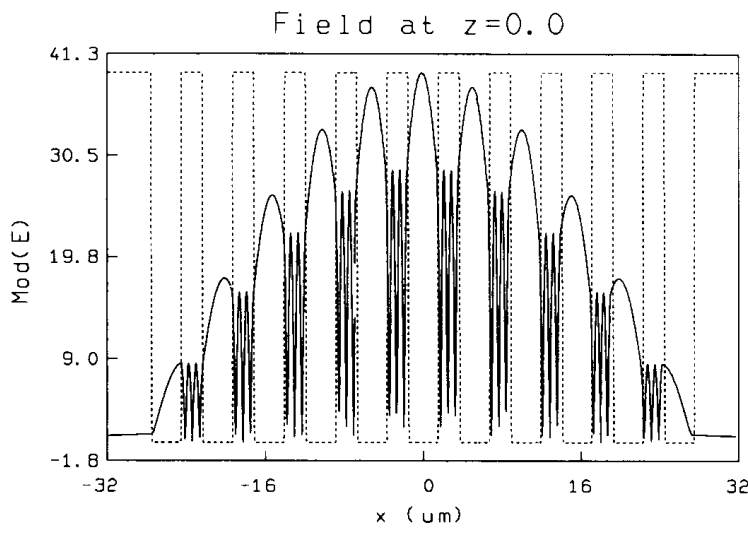

(a)

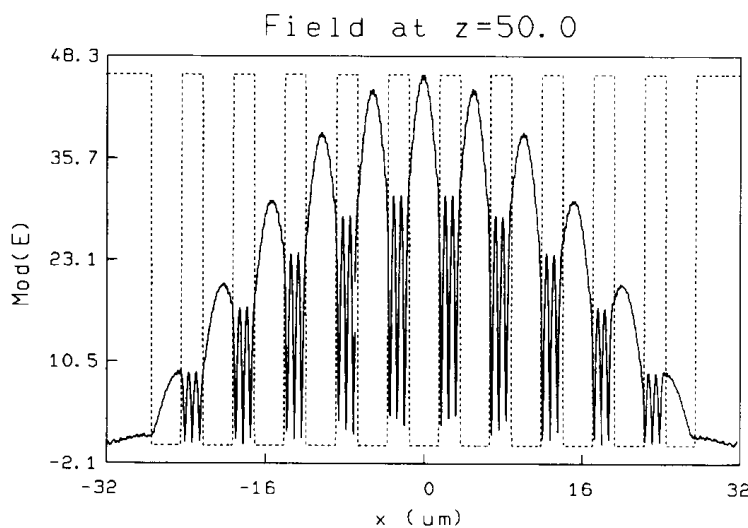

(b)

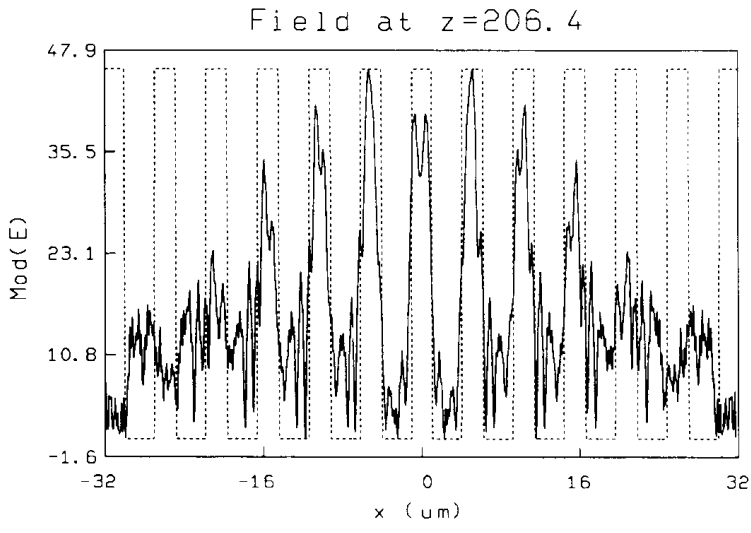

(c)

Fig. 6. Intensity profiles of (a) mode 11 at $z=0 \mu \mathrm{m}$, (b) mode 11 at $z=$ $50 \mu \mathrm{m}$, and (c) mode 11 at $z=206.4 \mu \mathrm{m}$. (The dashed lines represent the real refractive-index profile.)

\section{B. Talbot Filtering}

In order to analyze the filtering of the eleven array modes by the Talbot filter the field propagation of the modes in an array structure as shown in Fig. 2 was cal-

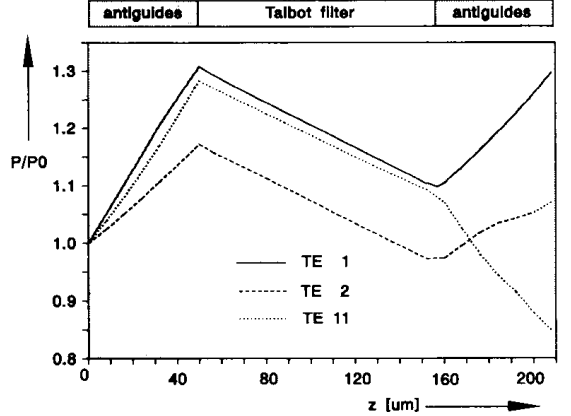

Fig. 7. Total power in the array structure versus $z$ for modes 1,2 , and 11 .

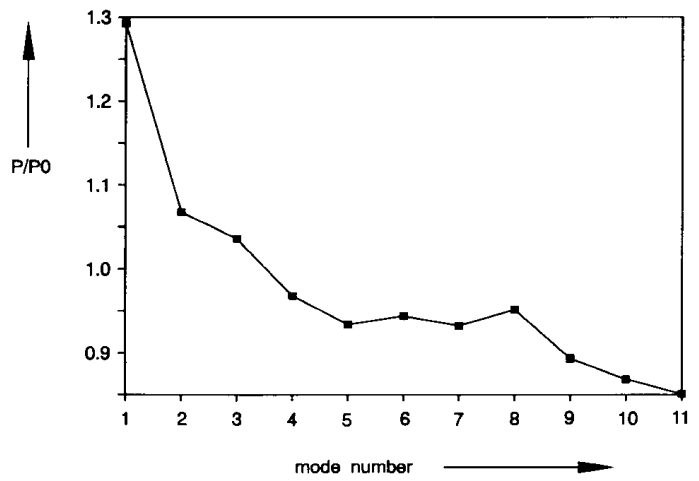

Fig. 8. Ratio $P / P_{0}$ of the total power $P$ and the input power $P_{0}$ in the array structure at $z=206.4 \mu \mathrm{m}$ for the eleven array modes.

culated using a program based on the beam-propagation method (BPM) [8]. The BPM is a computational tool widely used in integrated optics and consists of propagating a beam over a small distance through a homogeneous space followed by a correction on the refractive-index variations seen by the beam during the propagation step.

The array modes in a diode laser array are generally polarized parallel to the $x$ direction (Fig. 1) due to reflectances at the mirror facets. Although in our multilayer representation this would result in a TM-like polarization only the effect of the Talbot filter on TE polarized modes was calculated because the BPM does not allow for TM polarization.

Figs. 4, 5, and 6 display the intensity profiles of modes 1,2 , and 11 at the end of the first and the second set of antiguides. The profiles at the end of the first set of antiguides still resemble the initial ones for all three modes. However, at the end of the second set of waveguides modes 1 and 2 will still resemble the initial intensity profiles while the out-of-phase mode, mode 11, appears to be distorted. Most of its intensity is found in the interelement regions, characterized by high losses, which leads to the suppression of this mode.

The fact that the first two modes are not filtered out can also be inferred from Fig. 7, showing the ratio of the total 
power in the array structure $P$ and the input power $P_{0}$ verus $z$ for modes 1,2 , and 11 . Modes 1 and 2 show a power increase in both sets of antiguides, while mode eleven only shows an increase in power in the first set of antiguides. Fig. 8 displays the ratio $P / P_{0}$ at the end $(z=206.4 \mu \mathrm{m})$ of the total array structure for the eleven modes. It clearly shows that not only modes 2 and 11 but also modes 3 through 10 are filtered out effectively.

Although the second mode is not affected by the Talbot filter, which is in agreement with the findings of Botez et al. [5], it will be unlikely that the array will operate in this mode since the modal discrimination between modes 1 and 2 at and even close [10] to resonance is very high, as shown in Fig. 3, due to the fact that the second mode suffers more edge-diffraction losses than the in-phase mode [5]. Thus, the array will have a diffraction-limited output since modes 2 through 11 are either filtered out or are prevented from lasing due to a high modal loss.

\section{Conclusions}

An analysis of the Talbot filtering of all the array modes in an ROW array of antiguides has been demonstrated. It has been shown that an ROW array of antiguides with a Talbot filter is an effective means to achieve single inphase mode operation with a diffraction-limited output.

\section{ACKNOWLEDGMENT}

The authors gratefully acknowledge the valuable discussions with H. Martinot, F. Lozes-Dupuy, and H. J. W. M. Hoekstra.

\section{REFERENCES}

[1] D. Botez and D. E. Ackley, "Phase-locked arrays of semiconductor diode lasers," IEEE Circuits Device Mag., pp. 8-16, 1986.

[2] D. Botez, L. J. Mawst, G. Peterson, and T. J. Roth, "Resonant optical transmission and coupling in phase-locked diode laser arrays of antiguides: The resonant optical waveguide (ROW) array," Appl. Phys. Lett., vol. 54, no. 22, pp. 2183-2185, 1989.

[3] L. J. Mawst et al., "Phase-locked array of antiguided lasers with monolithic spatial filter," Electron. Lett., vol. 25 , no. 5 , pp. 365$366,1989$.

[4] L. J. Mawst, D. Botez, M. Jansen, T. J. Roth, and G. Peterson, "High-power, narrow single-lobe operation from 20-element phaselocked arrays of antiguides," Appl. Phys. Lett., vol. 55, no. 20, pp. 2060-2062, 1989

[5] D. Botez, L. J. Mawst, G. Peterson, and T. J. Roth, "Phase-locked arrays of antiguides: Modal content and discrimination," IEEE $J$. Quantum Electron., vol. 26, no. 3, pp. 482-495, 1990.

[6] J. Z. Wilcox et al., "Design considerations for diffraction-coupled arrays with monolithically integrated self-imaging cavities," Appl. Phys. Lett., vol. 54, no. 19, pp. 1848-1850, 1989.

[7] D. Botez and G. Peterson, "Modes of phase-locked diode-laser arrays of closely spaced antiguides,"' Electron. Lett., vol. 24, no. 16, pp. 1042-1044, 1988.

[8] J. Van Roey, J. van der Donk, and P. E. Lagasse, "Beam-propagation method: Analysis and assessment," J. Opt. Soc. of Amer., vol. 71 , no. 7 , pp. $803-810,1981$.

[9] S. Wolfram, Mathematica, A System For Doing Mathematics By Computer. New York: Addison-Wesley, 1989, p. 426.

[10] P. D. Van Eijk, "An AIGaAs/GaAs laser array of antiguides with a monolithically integrated Talbot cavity: Array modes and Talbot filtering," L.A.A.S. du C.N.R.S. internal report \#90093, pp. 43-51, 1990.

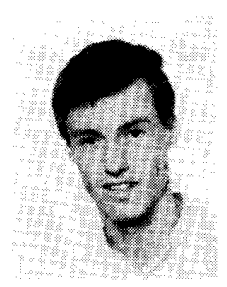

Pieter D. Van Eijk ( ${ }^{\prime}$ 87-M'89) was born in The Netherlands on December 4, 1966. He received the Dutch "docteraal" degree in electrical engineering from The University of Twente, Enschede, The Netherlands, in 1990. During his studies he gained professional experience through an internship at NASA Ames Research Center and at The Centre National de la Recherche Scientifique (CNRS) in Toulouse, France. He is currently working toward the M.S. degree in electrical engineering at UCLA. His main interests are integrated optics and optoelectronics.

*

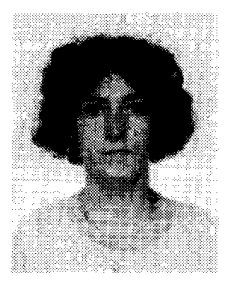

Muriel Reglat was born in Toulouse, France, in January 1963. She received the DEA (Diplome d'Etudes Approfondies) in electronics from the University Paul Sabatier of Toulouse in 1988. Currently she is working toward the Ph.D. degree at the device group of the Laboratoire d'Automatique et d'Analyse des Systèmes of the CNRS. Her main interests concern high-power laser diode arrays.

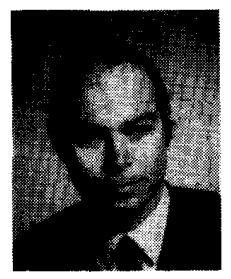

Georges Vassilieff was born in Toulouse, France, in January 1948. He received the M.S. degree in physics in 1968, the Ph.D. degree in 1971, and the Doctorat d'Etat en Sciences Physique in 1977. all from the University of Toulouse, France

Currently he is a Professor at the University of Toulouse and works at the Laboratoire d'Automatique et d'Analyse des Systèmes of the CNRS. in the device group. His research activities concern visible AlGaAs laser diodes and high-power laser arrays.

*

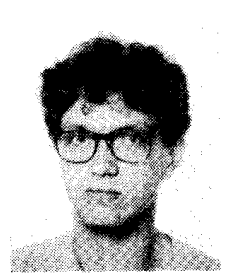

Gijs J. M. Krijnen (S'90) was born in The Netherlands, December 3, 1961. His Master Thesis, concerning magnetic recording, was carried out at Philips Laboratories in Eindhoven from March 1986 to March 1987. He received the M.Sc. degree in electrical engineering from The University of Twente, Enschede, the Netherlands, in 1987. His current Ph.D. work is concerned with integrated nonlinear optical switching devices.

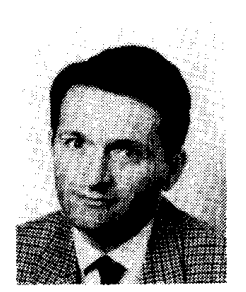

Alfred Driessen was born in 1949 in Grefrath, Germany. In 1972 he received a degree in physics, and in 1982 the $\mathrm{Ph} . \mathrm{D}$. degree, both from the University of Amsterdam.

From 1982 to 1987 he worked at the Physics Department of the Free University of Amsterdam on Hydrogen in metals and high temperature superconductors. Since 1988 he has been Group Leader of the Optical Group of the Subdepartment Transducers and Materials Science of the Physics Department of the University of Twente. His current research interest is mainly integrated optics for telecommunication.

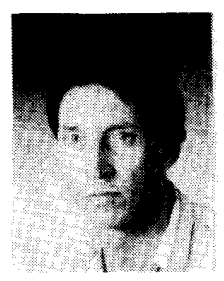

Anton J. Mouthaan (M'89) was born on July 10 1950 in the Hague. In 1973 he received the B.Sc degree in electrical engineering from The University of Twente, Enschede, The Netherlands, and the M.Sc. degree in 1976. Until 1977 he was a Research Assistant there, and in 1977 he became a Lecturer at the Department of Electrical Engineering at the University of Zambia. From 1982 to 1986 he was a Research Fellow at the IC-technology and Electronics Group in Twente and received the Ph.D. degree in 1986. In 1986 he be-

came a Senior Lecturer in Zambia and in 1988 he became an Associate Professor in Twente in the area of simulation and modeling, and CAD for analog circuits. 